\title{
Comparison of Unilateral versus Bilateral Kyphoplasty in Multiple Myeloma Patients and the Importance of Preoperative Planning
}

\author{
Ioannis D Papanastassiou ${ }^{1,2}$, Mohamed Eleraky ${ }^{1,3}$, Ryan Murtagh ${ }^{4}$, \\ Zinon T Kokkalis ${ }^{5}$, Maria Gerochristou ${ }^{6}$, Frank D Vrionis ${ }^{1}$

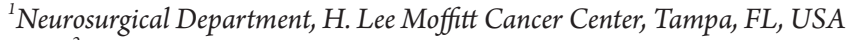 \\ ${ }^{2}$ General Oncological Hospital "Agioi Anargyroi", Athens, Greece \\ ${ }^{3}$ Neurosurgical Department, G.V. (Sonny) Montgomery VA Medical Center, Jackson, MS, USA \\ ${ }^{4}$ Radiology Department, H. Lee Moffitt Cancer Center, Tampa, FL, USA \\ ${ }^{5}$ First Department of Orthopaedics, Athens University Medical School, Athens, Greece \\ ${ }^{6}$ Department of Dermatology, "Andreas Syngros" Hospital, Athens, Greece
}

Study Design: Retrospective comparative study and technical note.

Purpose: To determine if there is a difference in clinical and radiographic parameters between unilateral and bilateral kyphoplasty in a uniform cancer population and to stress the importance of preoperative planning.

Overview of Literature: While unipedicular kyphoplasty is gaining popularity, a few comparative studies have reported on superior kyphotic reduction with the bipedicular approach.

Methods: We reviewed 69 myeloma patients with 105 operated levels (51 levels were done bilaterally vs. 54 unilaterally). Pain reduction, height restoration, cement volume and complications were recorded up to three months postoperatively. A technical note to identify the skin entry point on the basis of the magnetic resonance imaging and fluoroscopy (lateral view) is being described.

Results: Both procedures resulted in significant pain reduction (5.4-5.6/10 points, $p=0.8)$. There was significant height restoration after the operation $(p<0.001)$, while there was no sustained difference between the procedures $(p=0.5)$ up to three months postoperatively. More cement was injected in the bilateral group ( $4.1 \mathrm{~mL}$ vs. $4.9 \mathrm{~mL}, p=0.002)$; no difference in cement extravasation in the spinal canal was observed $(p=0.5)$.

Conclusions: There was no difference in the clinical or radiological outcomes between the unilateral and bilateral approaches. Therefore, unilateral kyphoplasty may be performed whenever it is technically feasible and this may be determined preoperatively.

Keywords: Compression fracture; Magnetic resonance imaging; Multiple myeloma; Kyphoplasty; Unilateral

\section{Introduction}

Vertebral compression fractures (VCF) constitute a major health problem affecting more than 1.4 million people each year worldwide, leading to pain, significant mor- bidity, and healthcare expenses [1]. Vertebroplasty (VP) and balloon kyphoplasty (BKP) are minimally invasive techniques that have been successfully employed to treat painful VCFs [2,3]. BKP pertains to the application of an inflatable balloon that creates a cavity before cement aug-

Received Apr 3, 2013; Revised Aug 2, 2013; Accepted Aug 10, 2013

Corresponding author: Ioannis D Papanastassiou

Neurosurgical Department, H. Lee Moffitt Cancer Center and Research Institute,

University of South Florida College of Medicine, 12902 Magnolia Drive, Tampa, Fl, 33647, USA

Tel: +1-813-745-4673, Fax: +1-813-745-3713, E-mail: jpapa73@yahoo.gr 
mentation and was introduced in the late 90s.

BKP has been traditionally performed bilaterally, especially in the lumbar spine. However, the unilateral approach is gaining popularity due to decreased surgical time, radiation exposure, costs and overall complication rate [4-11]. Whereas most authorities feel that both approaches yield similar results, a couple of the existing comparative studies reported the superiority of the bilateral approach in terms of kyphosis reduction [12-14], with one group reporting opposite results between the early [12] and late phases [14].

To resolve this controversy, we performed a retrospective comparative study of unilateral vs. bilateral BKP in a uniform cancer population (patients with multiple myeloma). The null hypothesis of our study was that both operations provide equal pain relief, height restoration and cement filling in the fractured vertebrae. We also analyzed the technical aspects of the unilateral procedure and stress the importance of preoperative planning (especially using preoperative magnetic resonance imaging [MRI]) for the execution of BKP in an easy, safe and quick fashion.

\section{Materials and Methods}

\section{Preoperative planning/surgical technique}

The desired trajectory is drawn in an axial image in the level of the pedicle of the fractured vertebrae (Fig. 1A). The entry point/ site of incision should lie at a distance from the midline, with this distance calculated by the intersection of the cannula trajectory (line $\gamma$ ) with the skin line (line a).

The insertion angle can also be estimated (angle $\omega$ ) and the surgeon may get an idea of the inclination of the Jamshidi needle. If the angle is $45^{\circ}$, as known by the geometry of the right triangle, in each step the trocar should move an equal distance in the anteroposterior (AP) and lateral (L) radiographs. As the angle diminishes (i.e., away from midline) the ratio between the L/AP increases (the trocar moves "faster" in the L plane than the AP plane).

Angle $\varphi$ (Fig. 1B) gives a good approximation of the inclination that the trocar should have at the sagittal level. It also provides good evidence for the inclination that the fluoroscopy C-arm should have for each vertebra ("true" AP view) to "square" the endplates and avoid the "parallax" phenomenon.

After prepping and draping in the usual fashion, we positioned the biplanar fluoroscopy. The fracture level was identified and then a dotted line parallel to the midline was drawn on the skin in the distance calculated from the MRI (in this case $\mathrm{a}=4.4 \mathrm{~cm}$ ) (Fig. 2). The exact entry point was then found from the lateral radiograph, in line with the dotted skin line. A blunt instrument was used to calculate the desired trajectory in the sagittal view. The tip of the instrument was seen on fluoroscopy (Fig. 3A),
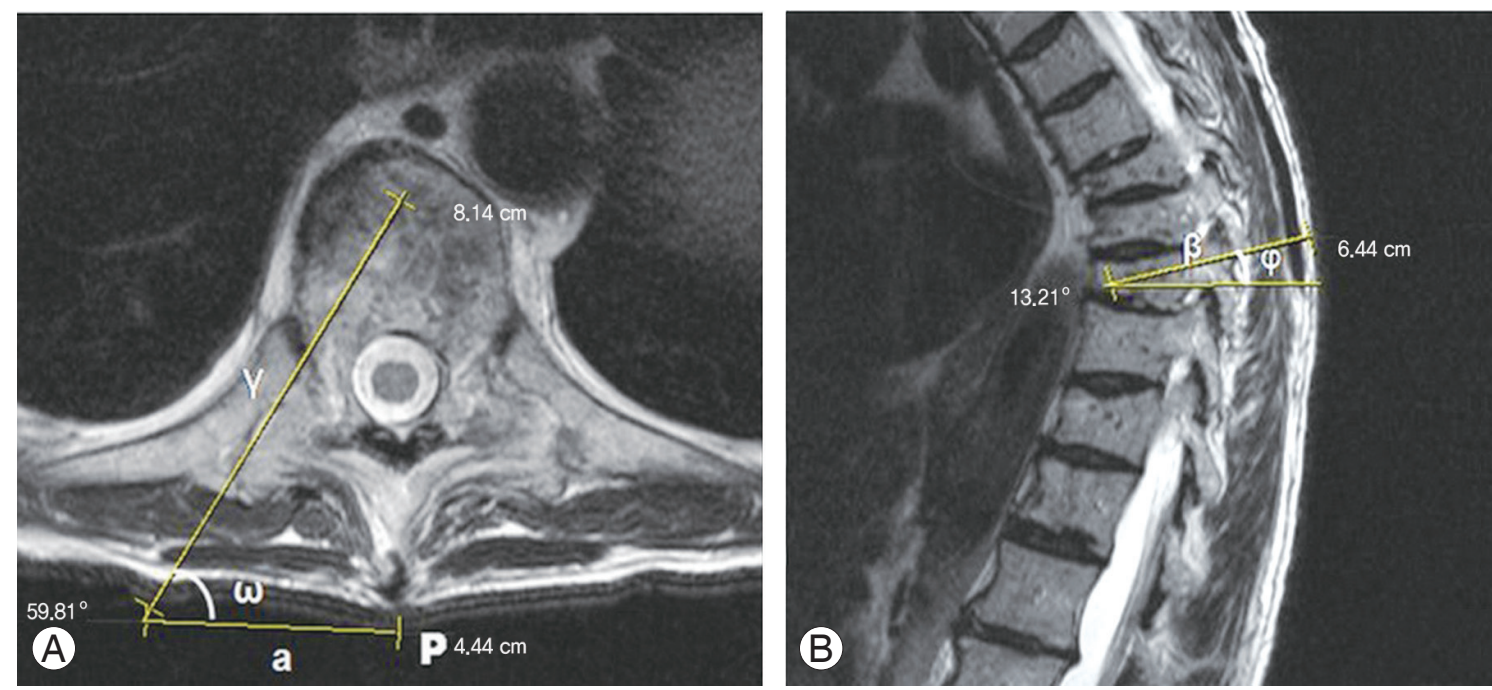

Fig. 1. (A) Preoperative planning: an axial magnetic resonance imaging (MRI) is chosen at the level of the pedicle to be cannulated. Line $\gamma$ represents the desired needle trajectory in the axial plane, line a the distance of the skin entry point (in this case 4.4 $\mathrm{cm}$ ) from the midline, and angle $\omega$ the insertion angle $\left(59.81^{\circ}\right)$. (B) Sagittal MRI at the level of the pedicle to be cannulated. Line $\beta$ represents the desired needle trajectory in the sagittal plane and angle $\varphi$ the inclination in the same plane $\left(13.21^{\circ}\right.$ in this case). 
and if it was felt to be in an optimal position, it served as our definitive entry point. We did not use the anteroposterior or oblique radiograph for localization of the entry point. Those were used later, as soon as the Jamshidi needle came in contact with the bone. The remainder of the procedure was performed as described thoroughly in the literature [15]; trocar insertion (Fig. 3B) and final radiographs after the same steps were repeated at each level as shown in Fig. 4.

Figs. 5-7 depict unilateral kyphoplasties in the lower lumbar spine.

We retrospectively reviewed 112 myeloma patients

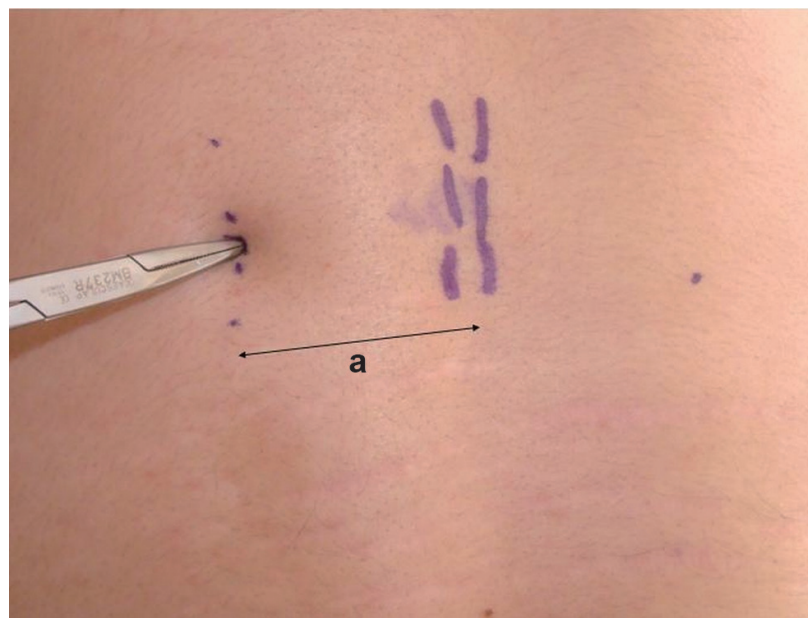

Fig. 2. The midline is marked by palpating the spinous processes. A parallel line (dotted line) is drawn in the distance calculated from the axial image (a); a blunt instrument is used for localization of the exact entry point (along the dotted line). who underwent 690 consecutive BKP procedures of the thoracolumbar spine (Kyphon Inc., Sunnyvale, CA, USA) in a single tertiary cancer center between October 2007 and August 2010. 410 levels were done in the thoracic and 280 levels in the lumbar spine, 310 through a bilateral and 380 with a unilateral procedure. Patient were candidates for cement augmentation if they had a VCF with at least $20 \%$ loss of anterior or middle vertebral body height and persistent pain not related to other causes as determined by the clinical exam, and imaging (e.g., discogenic or fascetogenic pain); the pain level should have been at least $4 / 10$ on a numerical scale, and not responsive for at least 2 weeks to conventional

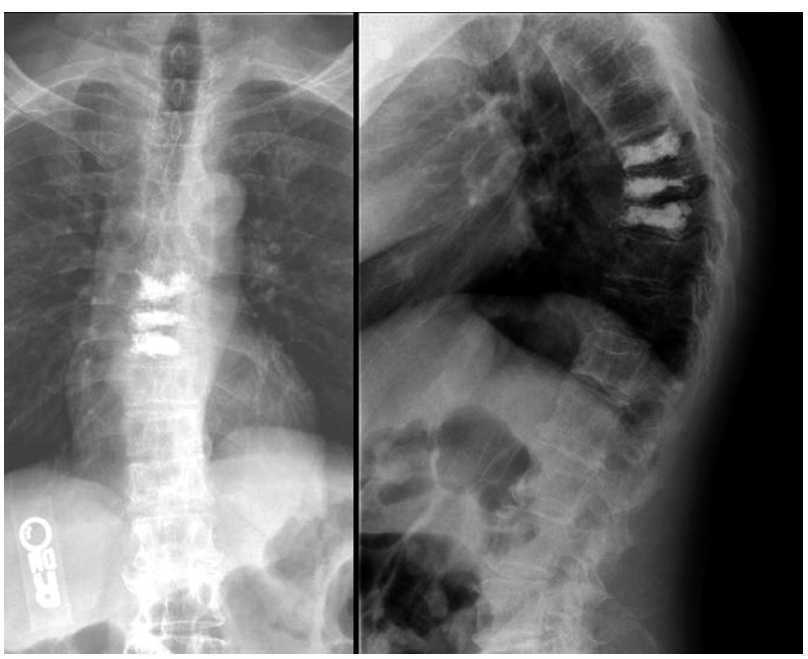

Fig. 4. Final radiographic outcome after the procedure was repeated at adjacent levels.
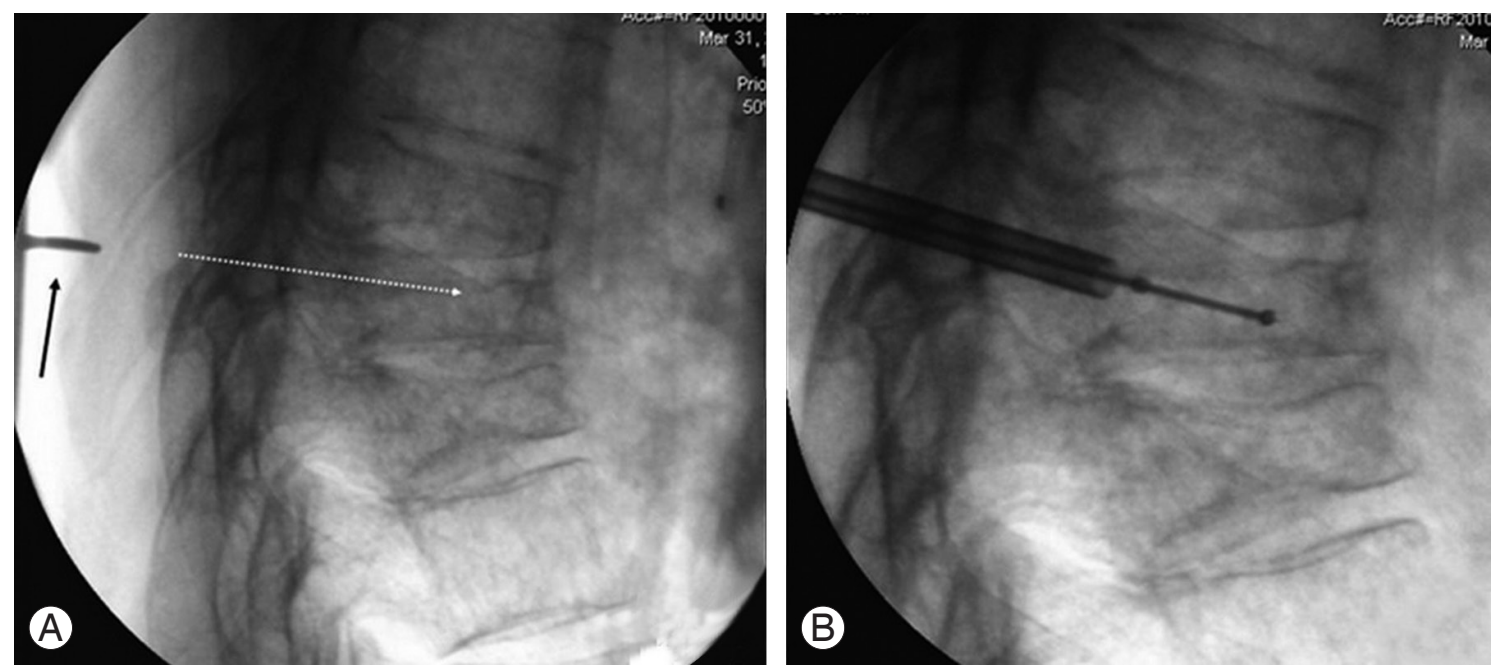

Fig. 3. (A) If the tip of the blunt instrument (black arrow) corresponds with the desired needle trajectory (white dotted line), it is marked as the final entry point. (B) Insertion of the working cannula/balloon. 
medical therapy, including narcotic analgesics, bracing, physical therapy, and bed rest. Symptoms had to be localized to the index vertebral body, and MRI (STIR sequence) was used to confirm the presence of edema in the fractured vertebra(e), which implied an acute or non healed state [16]. However, due to multi-level bone marrow involvement in the majority of cases, we frequently performed prophylactic augmentation in the adjacent vertebrae, especially in the thoracolumbar junction, to avoid subsequent fractures.

Inclusion criteria were: 1) acute or subacute fractures (fracture age up to 3 months, calculated from the time elapsed from the onset of symptoms up to the day of surgery), 2) satisfactory visualization of the end plates, 3) minimal follow up of three months, and 4) index level fracture with collapse and edema in the MRI. 69 patients met the inclusion criteria. $57 \%$ of the patients were males and the mean age was 61.6 years (range, $44-79$ years). 105 levels were augmented overall; in 51 levels a bilateral procedure was done vs. 54 levels unilaterally. In 36/69 patients both approaches were used ("hybrids"), while the remaining 33 patients underwent unilateral or bilateralonly procedures. Mean age in the unilateral group was $61.7( \pm 9.86)$ years vs. $63.7( \pm 9.44)$ years in the bilateral group ( $p=0.3$ ). The unilateral group contained 28 thoracic and 26 lumbar fractures, while the bilateral had 24 thoracic and 27 lumbar fractures.

Regarding procedure selection, we generally favored the unilateral approach in the thoracic spine; in the lum-

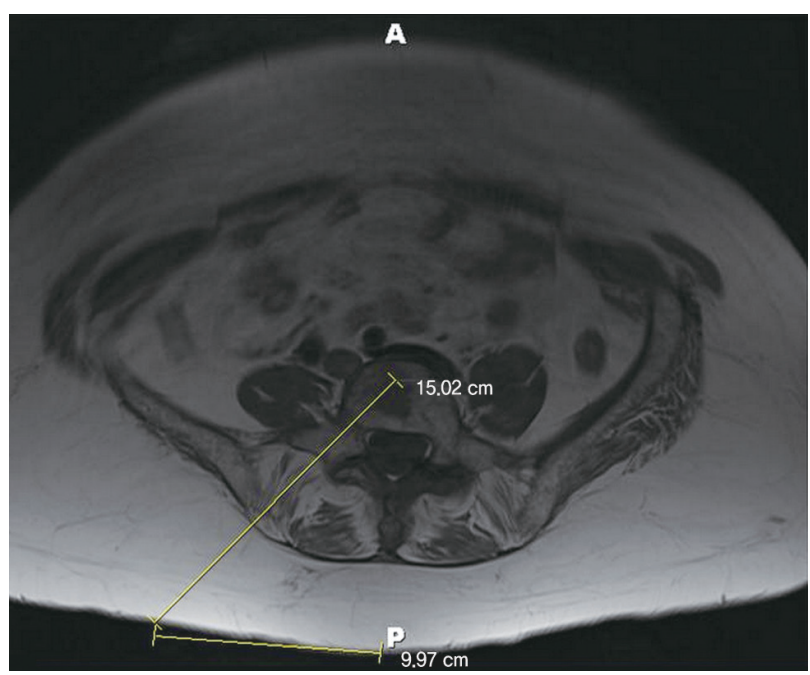

Fig. 5. Axial T1 magnetic resonance imaging at L5 level indicates the optimal trajectory and entry point (10 cm from midline). bar spine we preferred the unilateral approach whenever preoperative planning indicated it was safe and feasible (the trajectory reaching the midline at the middle or anterior third of the vertebral body without violation of the medial wall of the pedicle, such as is shown in Fig. 5). If the unilateral approach appeared hazardous preoperatively or the balloon was placed eccentrically or the cement did not cross the midline [17], we had a low threshold to go bilaterally. The same applied for severely collapsed thoracolumbar fractures.

The numerical pain scale $(0-10 ; 0$, no pain; 10 , worst imaginable pain) was compared before the operation and 3 months postoperatively, as retrieved from the medical records. This was done for the whole group, and also stratification was done according to the operation type. Vertebral height (anterior, middle, and posterior) was measured digitally (Emageon Inc., Birmingham, AL, USA) preoperatively, postoperatively and at three months using lateral radiographs of the spine (Fig. 8). This was

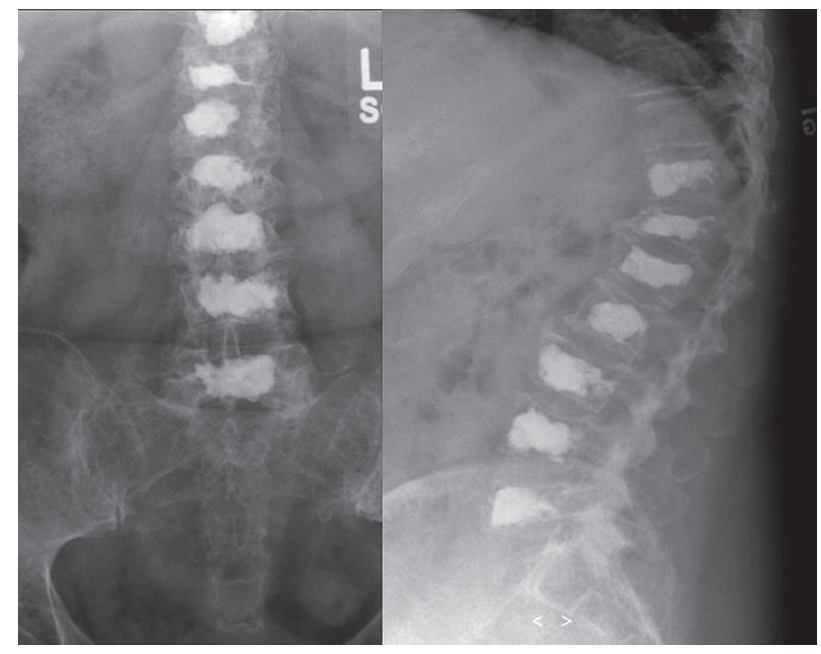

Fig. 6. Postoperative radiographs show satisfactory cement filling after unilateral operation in all levels.

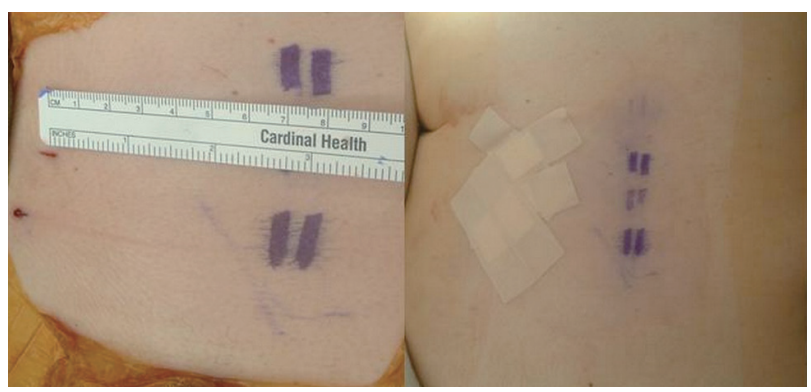

Fig. 7. Clinical image demonstrating the skin entry points. 
done simultaneously by the spine fellow (M.E.) and an experienced radiologist (R.M.). The absolute difference was reported in millimeters and compared between treatment groups. The amount of PMMA injected in each vertebral body was recorded from the intraoperative note. Finally, cement extravasation into the spinal canal was noted either from the patient's chart or postoperative radiographs, since it has been suggested that unilateral BKP may lead to a higher extravasation rate in this critical area $[12,13]$.

We used SPSS ver. 19.0 (IBM Co., Somers, NY, USA), for the statistical analysis. For pain ratings, height restoration and cement filling, we used the $t$-test, while for canal extravasation, we used Fisher's exact test. Results were considered significant at $p<0.05$.

\section{Results}

Mean pain level before the operation was 7.9 points and was reduced to 2.5 points 3 months postoperatively. This 5.4 point difference was statistically significant $(p<0.0005$, paired samples t-test) and should be considered also clinically relevant (more than $30 \%$ improvement from baseline pain) [18]. The mean pain improvement in the unilateral group was similar with the bilateral group (5.4 points vs. 5.5 points, $p=0.8$, independent-samples t-test). When we excluded patients that had hybrid procedures from the analysis, the same results were drawn (5.6 vs. 5.4, $p=0.8)$.

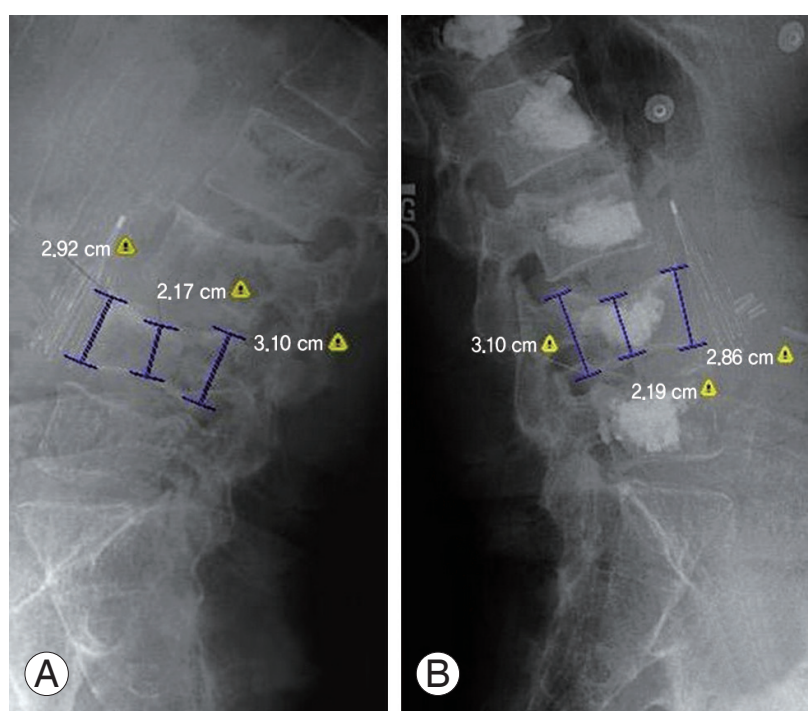

Fig. 8. (A, B) Measurements of the anterior, middle and posterior border of the vertebrae, before and after the procedure respectively.
The mean anterior height before the operation was $27.1 \mathrm{~mm}$ and was restored to $27.8 \mathrm{~mm}$ postoperatively $(p<0.0005$, paired samples t-test); this was sustained three months after the operation $(27.7 \mathrm{~mm}, p<0.0005)$. The eta squared statistic [19] indicated a large effect size (0.54). The middle height was $25 \mathrm{~mm}$ and was restored to 26.8 $\mathrm{mm}(p<0.0005)$ and $26.7 \mathrm{~mm}$ at 3 months, with a large size effect again (eta squared: 0.67 ). This depicts the fact that the majority of fractures were biconcave and most of the correction was done in the middle of the vertebra. Posterior height measurements were $27.9 \mathrm{~mm}, 28.1 \mathrm{~mm}$ $(p<0.0005)$ and $28 \mathrm{~mm}$ respectively. However, the magnitude of difference in the means $(1.4 \mathrm{~mm})$ indicated a moderate size effect (eta squared: 0.12 ).

The height restoration in the anterior border was $0.62 \mathrm{~mm}$ in the unilateral vs. $0.74 \mathrm{~mm}$ in the bilateral group (preoperative vs. postoperative, $p=0.3$, unpaired $t$-test). In addition, the magnitude of differences in the means $(1.2 \mathrm{~mm})$ was very small (eta squared: 0.009 ). In the middle of the vertebra where most of the height gain was encountered, values were $1.75 \mathrm{~mm}$ vs. $1.8 \mathrm{~mm}$ respectively ( $p=0.8$ ), with a negligible size effect $(0.5 \mathrm{~mm}$, eta squared $<0.0005)$. In the posterior border as expected there was minimal restoration $(0.1 \mathrm{~mm}$ vs. $0.19 \mathrm{~mm}, p=0.2)$. At the 3 -month interval, there was less than a $1 \mathrm{~mm}$ collapse (which was more pronounced in the middle aspect- $0.95 \mathrm{~mm}$ ), with minimal difference between the procedures.

The mean cement volume in the unilateral group was $4.1 \mathrm{~mL}$ vs. $4.9 \mathrm{~mL}$ in the bilateral $(p=0.002)$. The ratio of thoracic fracture to lumbar fracture was 1.07 in the unilateral vs. 0.88 in the bilateral group, although this was not significant (chi-square test with Yates continuity correction, $p=0.7$ ).

No serious complications were encountered in any of the cases. In $13.3 \%$ of the levels, cement extravasation was reported in the disk space and in $4.8 \%$ in the spinal canal. From the latter extravasations, 3 happened in the unilateral vs. 2 in the bilateral group ( $p=0.5$, Fisher's exact test); none was symptomatic. Results are summarized in Tables 1, 2.

\section{Discussion}

Traditionally, vertebral augmentation procedures (VAPs) have been described with bilateral pedicle cannulation $[15,20]$. However, gradually surgeons performed the pro- 
Table 1. Clinical and radiographic outcomes for the whole cohort

\begin{tabular}{lccc} 
Outcomes & Preoperative & Postoperative & 3 mo Postoperative \\
\hline Pain scale (0-10 points) & 7.9 & - & $2.5(p<0.0005)$ \\
Absolute height $(\mathrm{mm})$ & & & \\
\hline Anterior border & 27.1 & $27.8(p<0.0005)^{\mathrm{a}}$ & $27.7(p<0.0005)^{b)}$ \\
Middle & 25.0 & $26.8(p<0.0005)$ & $26.7(p<0.0005)$ \\
\hline Posterior border & 27.9 & $28.1(p<0.0005)$ & $28(p=0.08)$ \\
\hline
\end{tabular}

${ }^{\text {a) }} p$-value refers to preoperative-postoperative values comparison; ${ }^{\text {bl }} p$-value refers to preoperative-3 months postoperative values comparison.

Table 2. Comparison between the unilateral and bilateral groups

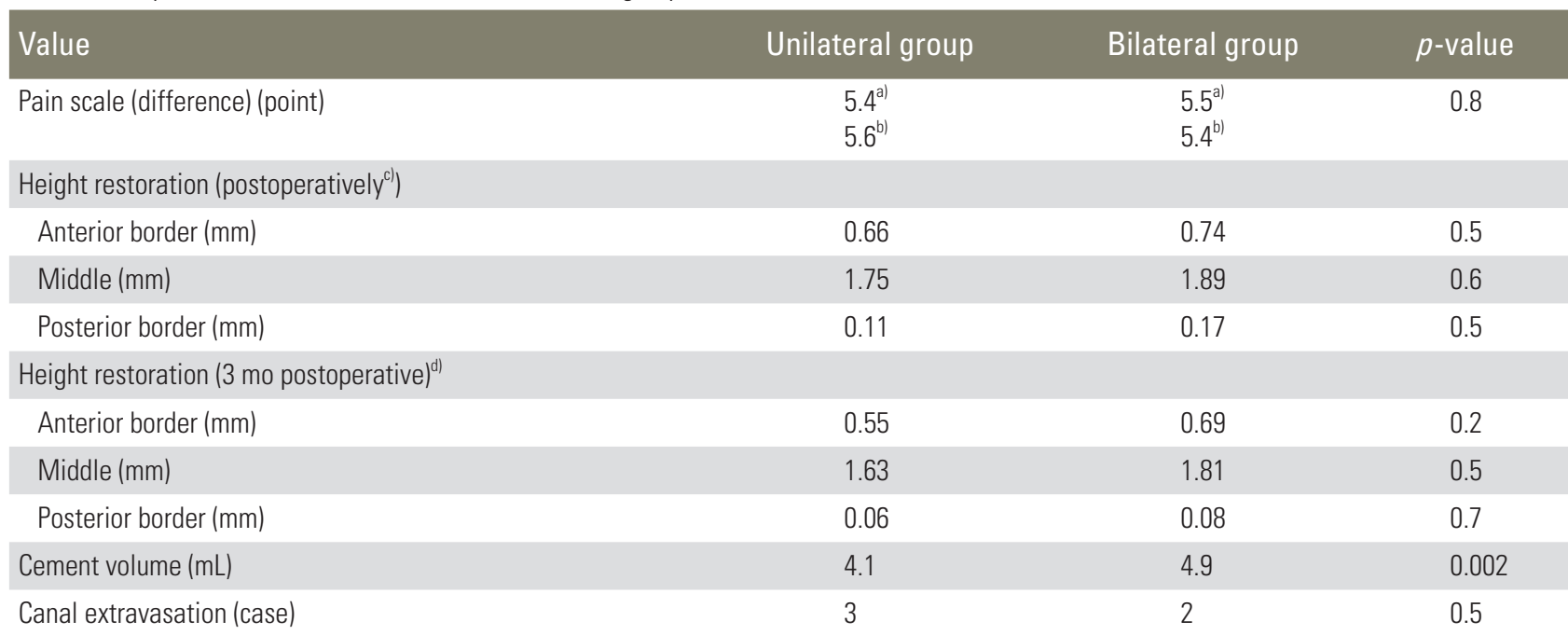

${ }^{\text {al }}$ Refers to all patients while ${ }^{\text {b) }}$ excludes patients with hybrid procedures; height restoration represents absolute values: ${ }^{\text {cl }}$ postoperative minus preoperative height and ${ }^{\mathrm{d}} 3$ months postoperative minus preoperative height.

cedure unilaterally, especially in the thoracic spine. The advantages are obvious: less radiation exposure, reduced cost, less operative time and potentially fewer complications. Also interpretation of fluoroscopic images is easier, since the introduction of the second cannula may obscure the field of vision. The majority of the studies in the literature, either biomechanical $[7,9,17]$ or clinical $[4-6,8,10]$, support its use and equivalent effectiveness with regard to the bilateral approach, with satisfactory cement filling and coronal alignment. However, few comparative studies have been published up to date; there are 4 studies on osteoporotic fractures [11-14,21] and 1 on cancer-related fractures [22]. This is the second and biggest comparative study in a uniform cancer population, the first being a small series of 19 levels overall [22]. Chen et al. [12] reported no difference in clinical outcomes; regarding height restoration, the authors found that the bipedicular group had more correction in the early postoperative period, whereas with longer observation (up to 2 years), both techniques yielded similar results [14]. Chung et al. [13] reported superior reduction in the kyphotic angle in the bilateral group, which was sustained until the last follow-up, although the pain reduction was similar between the groups. Song et al. [21] in a retrospective series reported no radiological differences although the unilateral group had more pain relief. In a more recent study, the authors claimed both approaches to be clinically and radiographically equivalent, except for the less radiation exposure and shorter surgical time in the unipedicular procedure [11]. Finally, in the small retrospective cancer cohort reported earlier, the authors found no clinical or radiological differences among the procedures, although they did report a trend favoring better kyphosis reduction in bipedicular kyphoplasty [22]. In our study, significant pain reduction and height restoration were observed after the operation, with the restoration more pronounced in the middle of the vertebra. We did not encounter a difference in clinical or radiological parameters between the 
treatment arms.

The only difference observed was the larger cement volume injected in the bilateral operation, in agreement with other published studies $[11,13,14]$. However, it is unknown if this difference in cement filling $(0.8 \mathrm{~mL})$ has clinical relevance, since the optimal amount of cement has not been determined; filling as low as $14 \%$ or $3.5 \mathrm{~mL}$ has been suggested as sufficient in restoring vertebral stiffness [23]. Additionally, the bigger ratio of thoracic to lumbar surgeries in the unilateral group influenced to a certain extent this difference (smaller cement volume in the thoracic spine). Again, the notion that if there is unsatisfactory filling (cement not crossing the midline [17]), one should have a low threshold to go on both sides should be emphasized. Finally, although studies failed to show lateral wedging after the unipedicular approach $[7,8]$, if the surgeon is doing multiple levels and feels there is uneven cement distribution, he may very well alternate sides.

A potential problem reported in the literature with unilateral kyphoplasty is cement extravasation in the spinal canal $[12,13,24]$. Due to the more medialized trajectory, the trocar gets closer to the spinal canal, so the surgeon should interpret radiographs carefully. Special attention should be paid if the balloon or cement goes backwards. If the cement goes backwards towards the canal, the operator should either wait until the cement hardens and then proceed very slowly or go to the contralateral side. In our study, there was no difference in canal extravasation between the groups, although we do recognize the higher propensity for canal extravasation in the unilateral technique.

The role of preoperative planning for executing VAPs has been underestimated, with most authorities making the incision site $1 \mathrm{~cm}$ lateral to the costotransverse junction in the thoracic spine and $1 \mathrm{~cm}$ lateral to the transverse process in the lumbar spine, as suggested by intraoperative fluoroscopy $[8,10]$. However, this simplistic approach does not take into account individual body habitus. Ryu et al. [10] reported a novel method to estimate the entry point: through 3 parallel lines in the $\mathrm{AP}$, an intersecting line is drawn in the lateral view with a constant dimension ratio. Whereas the technique is intriguing, we find it complex and difficult to apply in comparison with our method. Another interesting technical note was published by Chang et al. [6] in relation to the use of oblique fluoroscopy. The authors challenged the traditional $20^{\circ}$ to $30^{\circ}$ inclination of the C-arm suggested by others [5] and calculated the individual needle angle from an axial MRI. They also stressed the importance of accurate identification of the entry point and correct sagittal and axial plane angulation of the needle. It appears to be a good technique for surgeons who use a single $\mathrm{C}$-arm and are familiar with the oblique view. In a recent technical note of extrapedicular kyphoplasty, the authors also utilized MRI or computed tomography scanning to estimate the insertion angle and aimed the needle to the supero-lateral corner of the pedicle [24].

The unipedicular approach is almost always feasible in the thoracic spine via the costotransverse approach, as described early on by Brugieres et al. [25]. In the lumbar spine, the presence of descending lumbar nerve roots makes this technique more challenging [6]. However, with the approach we describe, in many cases it is feasible, with a more medialized direction starting from the mamillary process and aiming at the middle of the vertebral body, especially in the lower lumbar spine. Long and narrow pedicles, like in the mid-upper lumbar spine where the nerve roots lie closer to the vertebral body [26], may make its application more hazardous. Recent reports claim that even extrapedicular approaches may be feasible in the lumbar spine and suggest a safe extrapedicular zone, located between the junction of the body and the supero-lateral corner of the pedicle [24].

There are certain limitations in our study since this was a retrospective series and selection bias may apply. We generally opted for the unilateral procedure in the thoracic spine and whenever possible (as determined by the preoperative planning) in the lumbar spine. Second, interpretation of the clinical outcome (pain) may be confusing due to patients experiencing multiple fractures or having augmentation with both techniques (hybrids). We tried to ameliorate this problem by performing additional analysis, excluding those patients who had yielded similar results. Finally, we chose to compare only absolute height restoration and not kyphotic angle reduction or relative height restoration for several reasons: due to the predominance of biconcave fractures, the use of the kyphotic angle would underestimate the overall reduction; additionally, restoration relative to the initial height as calculated by adjacent vertebrae was not possible in many cases due to the presence of multiple adjacent fractures. Regarding our proposed technique, there are also some limitations: First, the MRI was performed with the pa- 
tient lying supine, while the procedure was performed while the patient was in the prone position. However, we have not found this to cause a significant discrepancy; plus our method does not pertain to millimeter accuracy. Second, sometimes thin cut axial images may not be available, missing the level of the pedicle or not being parallel to the superior endplate. Finally, a bipedicular approach or augmentation with curved curettes or newer cannula designs may make the need for exact estimation of the skin entry less mandatory.

Most of the fractures in our cohort of multiple myeloma patients were biconcave, depicting some of the distinctive features of this population, although similar morphology has occasionally been predominant in other osteoporotic series [27-29]. Similar to the observation of Pradhan et al. [27], most of the reduction happened in the middle of the vertebral body and unilateral kyphoplasty may have a benefit since it places the inflatable balloon more centrally in the body. In an osteoporotic patient with a solitary fracture, kyphosis reduction may be desirable and the bilateral procedure may have a theoretical advantage-although not proven as discussed above-without significantly delaying the operation. In the cancer setting, things may be totally different: multiple levels may need to be done in patients with significant co-morbidities where pain reduction is the only goal and kyphotic reduction may not apply. In this scenario, it is easy to understand why a unilateral approach may be preferable since it leads to significant reduction in the operative time and hazards of surgery, multiplied by the number of levels performed. Bilateral kyphoplasty may be preferable in cases of vertebra plana since on those occasions the lateral pillars of the vertebral body tend to be better preserved than the middle portion.

\section{Conclusions}

In our study there was no difference in the clinical or radiological outcome between the unilateral and bilateral approach. Therefore, in a cancer population, unilateral kyphoplasty should be performed whenever technically feasible-as determined by preoperative planning-without compromising results. We also present an easy and fast method for estimating the skin entry point in vertebral augmentation procedures. The role of preoperative MRI should be emphasized in planning/executing the procedure and identifying the patient's individual ana- tomic landmarks.

\section{Conflict of Interest}

Dr. Vrionis has received research grants and honoraria from Medtronic Inc. However, no funding has been received for the work presented in this paper. The other authors have nothing to disclose.

\section{References}

1. Johnell O, Kanis JA. An estimate of the worldwide prevalence and disability associated with osteoporotic fractures. Osteoporos Int 2006;17:1726-33.

2. Klazen CA, Lohle PN, de Vries J, et al. Vertebroplasty versus conservative treatment in acute osteoporotic vertebral compression fractures (Vertos II): an openlabel randomised trial. Lancet 2010;25:1085-92.

3. Wardlaw D, Cummings SR, Van Meirhaeghe J, et al. Efficacy and safety of balloon kyphoplasty compared with non-surgical care for vertebral compression fracture (FREE): a randomised controlled trial. Lancet 2009;373:1016-24.

4. Lee SB, Cho KS, Huh PW, et al. Clinical and radiographic results of unilateral transpedicular balloon kyphoplasty for the treatment of osteoporotic vertebral compression fractures. Acta Neurochir Suppl 2008;101:157-60.

5. Kim AK, Jensen ME, Dion JE, Schweickert PA, Kaufmann TJ, Kallmes DF. Unilateral transpedicular percutaneous vertebroplasty: initial experience. Radiology 2002;222:737-41.

6. Chang WS, Lee SH, Choi WG, Choi G, Jo BJ. Unipedicular vertebroplasty for osteoporotic compression fracture using an individualized needle insertion angle. Clin J Pain 2007;23:767-73.

7. Steinmann J, Tingey CT, Cruz G, Dai Q. Biomechanical comparison of unipedicular versus bipedicular kyphoplasty. Spine (Phila Pa 1976) 2005;30:201-5.

8. Papadopoulos EC, Edobor-Osula F, Gardner MJ, Shindle MK, Lane JM. Unipedicular balloon kyphoplasty for the treatment of osteoporotic vertebral compression fractures: early results. J Spinal Disord Tech 2008;21:589-96.

9. Tohmeh AG, Mathis JM, Fenton DC, Levine AM, Belkoff SM. Biomechanical efficacy of unipedicular versus bipedicular vertebroplasty for the manage- 
ment of osteoporotic compression fractures. Spine (Phila Pa 1976) 1999;24:1772-6.

10. Ryu KS, Park CK, Kim MK, Kim DH. Single balloon kyphoplasty using far-lateral extrapedicular approach: technical note and preliminary results. J Spinal Disord Tech 2007;20:392-8.

11. Wang Z, Wang G, Yang H. Comparison of unilateral versus bilateral balloon kyphoplasty for the treatment of osteoporotic vertebral compression fractures. J Clin Neurosci 2012;19:723-6.

12. Chen C, Chen L, Gu Y, et al. Kyphoplasty for chronic painful osteoporotic vertebral compression fractures via unipedicular versus bipedicular approachment: a comparative study in early stage. Injury 2010;41:3569.

13. Chung HJ, Chung KJ, Yoon HS, Kwon IH. Comparative study of balloon kyphoplasty with unilateral versus bilateral approach in osteoporotic vertebral compression fractures. Int Orthop 2008;32:817-20.

14. Chen C, Wei H, Zhang W, et al. Comparative study of kyphoplasty for chronic painful osteoporotic vertebral compression fractures via unipedicular versus nipedicular approach. J Spinal Disord Tech 2011; 24:E62-5.

15. Lieberman IH, Dudeney S, Reinhardt MK, Bell G. Initial outcome and efficacy of "kyphoplasty" in the treatment of painful osteoporotic vertebral compression fractures. Spine (Phila Pa 1976) 2001;26:1631-8.

16. Meyers SP, Wiener SN. Magnetic resonance imaging features of fractures using the short tau inversion recovery (STIR) sequence: correlation with radiographic findings. Skeletal Radiol 1991;20:499-507.

17. Chen B, Li Y, Xie D, Yang X, Zheng Z. Comparison of unipedicular and bipedicular kyphoplasty on the stiffness and biomechanical balance of compression fractured vertebrae. Eur Spine J 2011;20:1272-80.

18. Carragee EJ, Cheng I. Minimum acceptable outcomes after lumbar spinal fusion. Spine J 2010;10:313-20.

19. Olejnik S, Algina J. Generalized eta and omega squared statistics: measures of effect size for some common research designs. Psychol Methods 2003;8:434-47.

20. Deramond H, Depriester C, Galibert P, Le Gars D.
Percutaneous vertebroplasty with polymethylmethacrylate. Technique, indications, and results. Radiol Clin North Am 1998;36:533-46.

21. Song BK, Eun JP, Oh YM. Clinical and radiological comparison of unipedicular versus bipedicular balloon kyphoplasty for the treatment of vertebral compression fractures. Osteoporos Int 2009;20:1717-23.

22. La Maida GA, Sala F, Callea G, Capitani D, Singh S. Efficacy of unipedicular baloon kyphoplasty for treatment of multiple myeloma vertebral lesions. Asian Spine J 2011;5:162-8.

23. Liebschner MA, Rosenberg WS, Keaveny TM. Effects of bone cement volume and distribution on vertebral stiffness after vertebroplasty. Spine (Phila Pa 1976) 2001;26:1547-54.

24. Cho SM, Nam YS, Cho BM, Lee SY, Oh SM, Kim MK. Unilateral extrapedicular vertebroplasty and kyphoplasty in lumbar compression fractures: technique, anatomy and preliminary results. J Korean Neurosurg Soc 2011;49:273-7.

25. Brugieres P, Gaston A, Heran F, Voisin MC, Marsault C. Percutaneous biopsies of the thoracic spine under CT guidance: transcostovertebral approach. J Comput Assist Tomogr 1990;14:446-8.

26. Uribe JS, Arredondo N, Dakwar E, Vale FL. Defining the safe working zones using the minimally invasive lateral retroperitoneal transpsoas approach: an anatomical study. J Neurosurg Spine 2010;13:260-6.

27. Pradhan BB, Bae HW, Kropf MA, Patel VV, Delamarter RB. Kyphoplasty reduction of osteoporotic vertebral compression fractures: correction of local kyphosis versus overall sagittal alignment. Spine (Phila Pa 1976) 2006;31:435-41.

28. Kasperk C, Hillmeier J, Noldge G, et al. Treatment of painful vertebral fractures by kyphoplasty in patients with primary osteoporosis: a prospective nonrandomized controlled study. J Bone Miner Res 2005;20:604-12.

29. Rollinghoff M, Siewe J, Zarghooni K, et al. Effectiveness, security and height restoration on fresh compression fractures: a comparative prospective study of vertebroplasty and kyphoplasty. Minim Invasive Neurosurg 2009;52:233-7. 